\title{
Dynamics of tsetse natural infection rates in the Mouhoun river, Burkina Faso, in relation with environmental factors
}

\author{
Jérémy Bouyer ${ }^{1,2,3 *}$, Naférima Koné ${ }^{4}$ and Zakaria Bengaly 4 \\ 1 Unité Mixte de Recherche Contrôle des Maladies Animales Exotiques et Emergentes, Centre de Coopération Internationale en Recherche Agronomique pour le \\ Développement, Montpellier, France \\ 2 Unité Mixte de Recherche 1309 Contrôle des Maladies Animales Exotiques et Emergentes, Institut National de la Recherche Agronomique, Montpellier, France \\ ${ }^{3}$ Service de Bio Ecologie et Pathologies Parasitaires, Laboratoire National d'Elevage et de Recherches Vétérinaires, Institut Sénégalais de Recherches Agricoles, \\ Dakar, Sénégal \\ ${ }^{4}$ Centre International de Recherche-Développement sur I'Elevage en Zone subhumide, Bobo-Dioulasso, Burkina Faso
}

\section{Edited by:}

Brice Rotureau, Institut Pasteur,

France

\section{Reviewed by:}

Marcotty Tanguy, Institute of Tropical Medicine, Belgium

Antje Hoppenheit, Institute of

Parasitology and Tropical Veterinary

Medicine, Germany

\section{*Correspondence:}

Jérémy Bouyer, Laboratoire National d'Elevage et de Recherches

Vétérinaires, Institut Sénégalais de Recherches Agricoles, BP2057

Dakar-Hann, Sénégal

e-mail: bouyer@cirad.fr
In Burkina Faso, the cyclical vectors of African animal trypanosomoses (AAT) are riverine tsetse species, namely Glossina palpalis gambiensis Vanderplank (G.p.g.) and Glossina tachinoides Westwood (G.t.) (Diptera: Glossinidae). Experimental work demonstrated that environmental stress can increase the sensitivity of tsetse to trypanosome infection. Seasonal variations of the tsetse infection rates were monitored monthly over 17 months (May 2006-September 2007) in two sites (Douroula and Kadomba). In total, 1423 flies were dissected and the infection of the proboscis, middle intestine and salivary glands was noted. All the positive organs were analyzed using monospecific polymerase chain reaction (PCR) primers. To investigate the role of different environmental factors, fly infection rates were analyzed using generalized linear mixed binomial models using the species, sex, and monthly averages of the maximum, minimum and mean daily temperatures, rainfalls, Land Surface Temperature day (LSTd) and night (LSTn) as fixed effects and the trap position as a random effect. The overall infection rate was $10 \%$ from which the predominant species was T. congolense (7.6\% of the flies), followed by T. vivax (2.2\% of the flies). The best model (lowest AICc) for the global infection rates was the one with the maximum daily temperature only as fixed effect $(p<0.001)$. For $T$. congolense, the best model was the one with the tsetse species, sex, maximum daily temperature and rainfalls as fixed effect, where the maximum daily temperature was the main effect $(p<0.001)$. The number of $T$. vivax infections was too low to allow the models to converge. The maturation rate of $T$. congolense was very high $(94 \%)$, and G. $t$. harbored a higher maturation rate $(p=0.03)$. The results are discussed in view of former laboratory studies showing that temperature stress can increase the susceptibility of tsetse to trypanosomes, as well as the possibility to improve AAT risk mapping using satellite images.

Keywords: vector competence, vector capacity, environmental stress, parasite extrinsic cycle, infection rate, maturation rate, temperature

\section{INTRODUCTION}

In sub-Saharan Africa, African animal trypanosomoses (AAT), transmitted by tsetse flies (genus Glossina), are among the main constraints to the development of cattle farming (Itard et al., 2003). Demographic and climatic pressures have modified tsetse habitats, which are more and more fragmented (Van den Bossche et al., 2010). In the Mouhoun river basin, Burkina Faso, the cyclical vectors of AAT are riverine tsetse species, namely Glossina palpalis gambiensis Vanderplank (G.p.g.) and Glossina tachinoides Westwood (G. t.) (Diptera: Glossinidae). The fragmentation of tsetse habitats was studied repeatedly in this area, in order to understand its impact on tsetse distribution and densities (Bouyer et al., 2005; Guerrini et al., 2008), population structure and dispersal (Bouyer et al., 2007, 2009; Koné et al., 2011a).

The risk of animal trypanosomoses was also mapped, based on the number of infectious fly per trap per day as a risk indicator (Bouyer et al., 2006; Guerrini and Bouyer, 2007). This risk mapping revealed that the most dangerous river sections were located at the border of protected forests, due to a "border effect" leading to increased tsetse densities. No difference was, however, observed between the tsetse infection rates in the different landscapes and no environmental factor could be associated to this infection rate.

The goal of the present study was to explore the relationship between tsetse infection rate and various environmental factors at a local scale (the Mouhoun river basin).

\section{MATERIALS AND METHODS ENTOMOLOGICAL SURVEY}

Seasonal variations of the tsetse infection rates were monitored monthly over 17 months (May 2006-September 2007) in two sites, Douroula $\left(12^{\circ} 36^{\prime} 06^{\prime \prime} \mathrm{N}, 03^{\circ} 16^{\prime} 54^{\prime \prime} \mathrm{W}\right)$ and Kadomba 
$\left(11^{\circ} 32^{\prime} 19^{\prime \prime} \mathrm{N}, 03^{\circ} 58^{\prime} 24^{\prime \prime} \mathrm{W}\right)$, using 20 and 13 biconical traps, respectively (Figure 1).

These sites were previously characterized regarding vegetation and tsetse population dynamics, and correspond to border of protected forests, with a high risk for AAT transmission, corresponding to the interface AAT cycle (Van den Bossche et al., 2010). Douroula harbors an open Sudanean gallery forest which is the preferred habitat of G. $t$. ( $85 \%$ of the captures) whereas Kadomba harbors a closed Guinean gallery forest which is the preferred habitat of G.p.g (99.96\% of the captures) (Koné et al., 2011b). The two habitats are comparable in terms of host availability: domestic animals are largely predominant, particularly cattle and small ruminants. Intensive poaching has destroyed all wild mammals, with the exception of Tragelaphus scriptus and Cephalophus rufilatus, present in very low densities (Bouyer et al., 2005). Reptiles are still abundant, and represented mainly by Varanus niloticus and Crocodylus niloticus. In total, 1423 flies were dissected (94 G.p.g and 534 G. t. in Douroula, and 795 G.p.g in Kadomba), and the infection of the proboscis, salivary glands and middle intestine was noted (the organs were dissected in this order). When at least one of the organs was positive, all the three organs of a given tsetse were analyzed using monospecific polymerase chain reaction (PCR) primers for Trypanosoma brucei sensu lato, T. congolense savannah type only and $T$. vivax (Solano et al., 1999; Desquesnes and Davila, 2002). For T. congolense, flies presenting an infected proboscis were considered as mature infections.

\section{ENVIRONMENTAL DATA}

The maximum, minimum and mean daily temperatures and rainfalls were obtained from meteorological stations located at 25 and $50 \mathrm{~km}$ from the rivers sections monitored in Douroula and Kadomba, respectively.

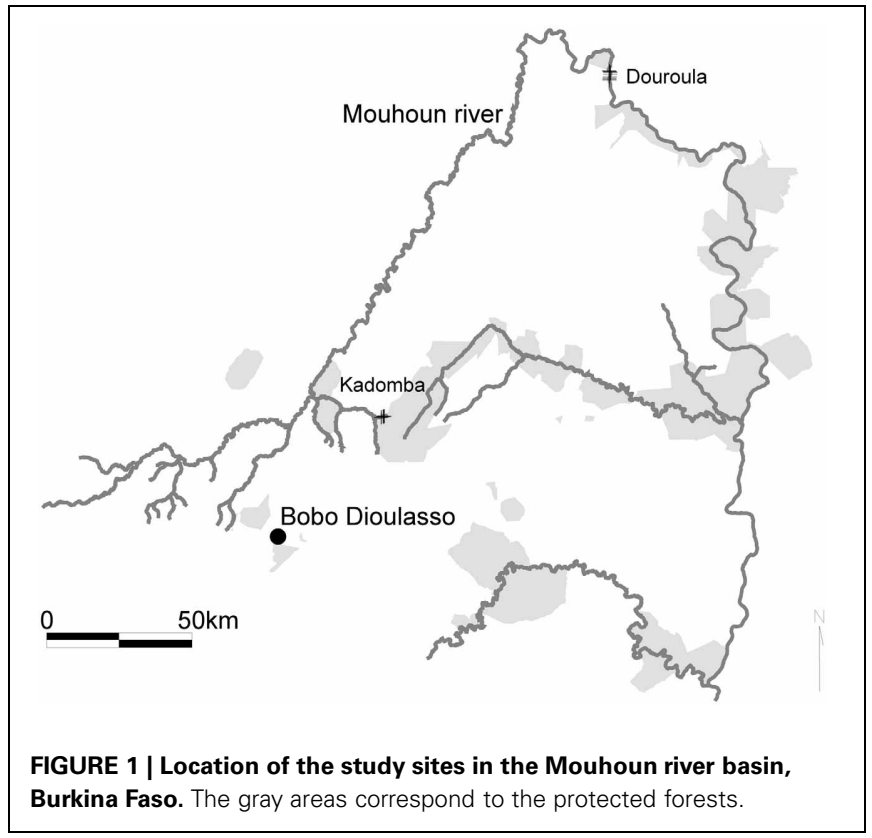

In addition, daily and nightly Land Surface Temperatures (LSTd and LSTn, respectively) measured from MODIS satellites were used as monthly average of the values in the $1^{*} 1 \mathrm{~km}$ pixels intersecting the sites where traps were set.

\section{STATISTICAL ANALYSES}

The fly infection rates were analyzed using generalized linear mixed binomial models (Laird and Ware, 1982) using the species, sex, and monthly averages of the maximum, minimum and mean daily temperatures, rainfalls, LSTd and LSTn as fixed effects and the trap position as a random effect. The best model was considered as the one with the lowest corrected Akaike information criterion (AICc) (Hurvich and Tsai, 1995; Burnham and Anderson, 2002).

The R software (R Core Team, 2013) was used for statistical analysis, together with the lme4 package for the linear mixedeffect model (Bates et al., 2011) and the MuMin package for the implementation of the AICc (Burnham and Anderson, 2002).

\section{RESULTS}

\section{INFECTION RATES}

The overall infection rate was $10 \%$ from which the predominant species was $T$. congolense (7.6\% of the flies), followed by T. vivax (2.2\% of the flies). For the remaining infected flies, the trypanosome species could not be identified and T. brucei was not observed.

The best model for the global infection rates was the one with the maximum daily temperature only as fixed effect $(p<0.001)$. The sex, age and other environmental variables did not improve the model predictions. The plot of cubic spline functions of environmental variables shows that the best relationship is actually observed for the maximum air temperature (Figure 2). LSTd had a similar relationship, although the reduction of the extent illustrates the weaker relationship. The increased confidence rate at low mean temperatures probably illustrated the intervention of
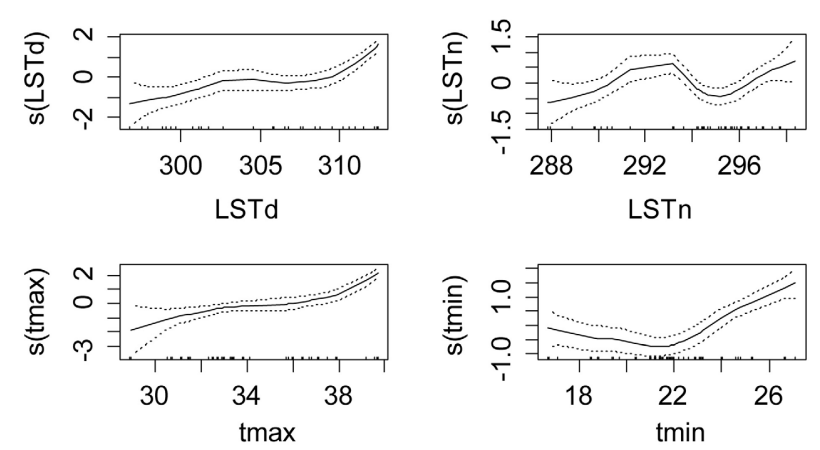

FIGURE 2 | Shape and amplitude of the relationships between tsetse infection rate and environmental variables. The values were estimated by a logistic regression model of cubic spline functions of environmental variables (LSTd and LSTn: MODIS Land Surface Temperature day and night in kelvins, tmin and tmax: minimum and maximum monthly averages of air temperature in ${ }^{\circ} \mathrm{C}$ measured in meteorological stations). For each

environmental variable $\mathrm{y}, \mathrm{s}(\mathrm{y})$ represents the fit of the regression model and the uncertainty lines present the standard error. 
other factors, like nutritional stress or others. LSTn and the minimum temperature did not present linear relationship with the infection rate.

For $T$. congolense, the best model was the one with the tsetse species, sex, maximum daily temperature and rainfalls as fixed effect, where the maximum daily temperature was the main effect $(p<0.001)$ and the sex and rainfalls had marginal effects on the infection rate (negative for females and for rainfalls). Table 1 presents the fixed coefficients of the best model. A similar but stronger relationship was observed between the maximum air temperature and the infection rate (Figure 3 ). Monthly rainfalls had a weaker inverse relationship with the infection rate.

The number of T. vivax infections was too low to allow the models to converge.

\section{MATURATION RATES}

The maturation rate of $T$. congolense was very high (94\%). The best model was the one with tsetse species, sex and maximum daily temperature as fixed effects, and only the species effect was significant, $G$. tachinoides having a higher maturation rate $(99 \%$, s.d. $1 \%)$ than G. p. gambiensis (92\%, s.d. $5 \%, p=0.03)$.

\section{DISCUSSION}

Trypanosomosis is also an infection for tsetse, which in turn try to control it through pre-existing defenses and a immune gene expression that is induced upon feeding on trypanosomes (Dyer et al., 2013 \# 1145). Many studies of vector manipulation by parasites demonstrated that parasites can impact vector survival, trophic behavior, fecundity (Lefevre and Thomas, 2008). Studies conducted in experimental conditions revealed that nutritional stress can increase their susceptibility to infection by trypanosomes (Akoda, 2009). For example, starvation increased the infection rates of Glossina morsitans morsitans Westwood with Trypanosoma congolense and the maturation rate of T. brucei brucei infections (Kubi et al., 2006). It was thereafter demonstrated that fly starvation can lead to a decreased expression of immune genes in newly hatched flies or a lack of immune responsiveness to trypanosomes in older flies, that can contribute to increased susceptibility of nutritionally stressed tsetse flies to trypanosome infection (Akoda et al., 2009b). In the case of tsetse infections with Trypanosoma brucei brucei,

Table 1 | Fixed effects of the best prediction model for $T$. congolense infection rates.

\begin{tabular}{lrlrl}
\hline Parameter & Estimate & Std. Error & z-value & $\operatorname{Pr}(>|\mathbf{z}|)$ \\
\hline (Intercept) & -15.32 & 1.98 & -7.75 & $9.09 e-15^{* * *}$ \\
G. tachinoides & 0.30 & 0.24 & 1.25 & 0.21 \\
Female & -0.39 & 0.22 & -1.80 & 0.0712. \\
tmax & 0.36 & 0.05 & 6.84 & $8.12 \mathrm{e}-12$. \\
Rainfalls & -0.005 & 0.002 & -1.96 & 0.0501.
\end{tabular}

Definition of the parameters: tmax, maximum monthly averages of air temperature in ${ }^{\circ} \mathrm{C}$ measured in meteorological stations; rainfalls: total monthly rainfalls in $\mathrm{mm}$.

Significance codes: $0^{* *} ; 0.05$. nutritional stress can also result in enhanced maturation of the trypanosome infection (Akoda et al., 2009a). It was also demonstrated that exposure of adult flies to high temperatures can increase the development rate of trypanosomes in adult flies (Leak, 1998). Moreover, flies emerging from pupae exposed to high temperatures are also more sensitive to infections by $T$. b. rhodesiense (Burtt, 1946) and T. congolense (Ndegwa et al., 1992). The impact of heat stress on tsetse immunity might thus be one of the explanations for the pattern observed in this study.

Under field conditions, a positive correlation was also observed between latitude, associated to increasing mean annual temperature and tsetse infection rates (Ford and Leggate, 1961). The authors stated that "at higher altitudes or, as in Zululand, a situation approaching the temperate zone, low infections occur as a response to cooler climates." In that study, the regression of proboscis-type infections on mean annual temperature was much more marked than that of gut and/or gut and proboscis-type infections, which is surprising given that a decreased immune response associated to heat stress is probably involved.

In our study, we observed a similar pattern at a more local scale and between seasons, i.e., a strong increase of infection rates with mean monthly maximum temperature. Moreover, the regression of all infections together on mean monthly temperature was less marked than for that of gut and/or gut and proboscis-type infections ( $T$. congolense). Since we used monthly average temperatures, both exposure of the pupae and the adult flies could have contributed to the observed pattern. Cattle rearing systems and farmer habits have been characterized in the study sites (Koné et al., 2012), together with a monitoring of AAT prevalence in sentinel herds (Métras et al., 2008). The sociological study showed that herders, particularly those of the Fulani ethnic group, tend to avoid the main river during the rainy season, when temporary ponds are available. Tsetse are, however, able to disperse
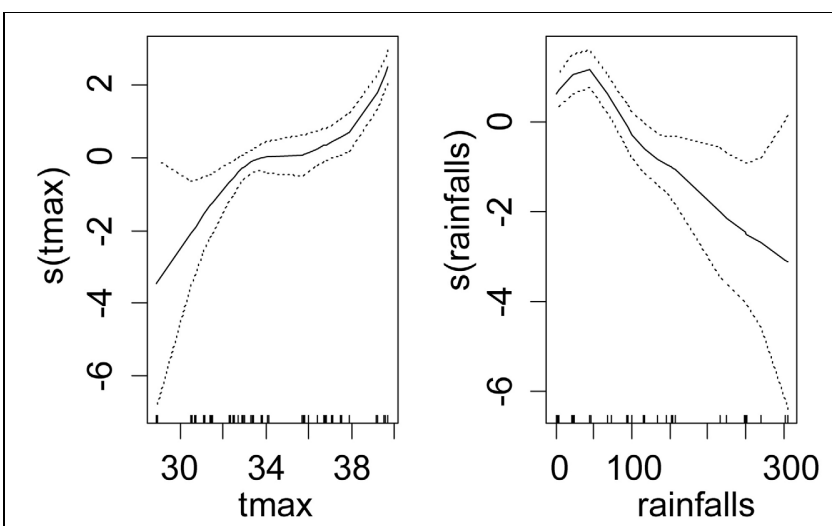

FIGURE 3 | Shape and amplitude of the relationships between tsetse infection rate with $T$. congolense and environmental variables. The values were estimated by a logistic regression model of cubic spline functions of environmental variables (tmax: maximum monthly averages of air temperature in ${ }^{\circ} \mathrm{C}$ measured in meteorological stations, rainfalls: total monthly rainfalls in $\mathrm{mm}$ ). For each environmental variable $\mathrm{y}, \mathrm{s}(\mathrm{y})$ represents the fit of the regression model and the uncertainty lines present the standard error. 
outside the gallery forest during this season, up to $2 \mathrm{~km}$ in the neighboring savannah (Cuisance et al., 1985; Vreysen et al., 2013). Overall, the AAT infection rates in cattle were similar between sites but showed a significant peak in July (middle of the rainy season), which is different from the peak of infection observed in tsetse (hot dry season, from April to May, when temperatures were highest). It is thus not likely that the pattern observed in this study was driven by an increased contact with infected hosts and this indirect effect would not give such a strong signal.

The impact of temperature on the maturation rate of $T$. congolense was not significant, which might be due to overall very high maturation rates observed. This maturation rate might have been overestimated by considering any fly positive in its proboscis as a mature infection.

Mapping vectorial diseases rely on mapping different risk indicators, like the vectorial capacity (Tran et al., 2005). The main elements of the vectorial capacity are the relative density of vectors to hosts, the survival of vectors, their feeding preferences and their infectious rate. Recent AAT risk modeling efforts based on satellite images in the Mouhoun river mainly relied on mapping tsetse catches per trap per day, which are considered to be correlated to their relative densities to the hosts (Bouyer et al., 2006; Guerrini and Bouyer, 2007). Even if the number of infectious fly per trap per day were considered, corresponding to the entomological inoculation rate, an indicator commonly used to map malaria risk for example, no environmental predictor of tsetse infection rate was evidenced. The integration of the results presented here in these AAT mapping efforts would probably lead to improved accuracies of the maps, and since the densities of tsetse are relatively stable over time ( $\mathrm{k}$ demographic strategy) (Koné et al., 2011b), it might also allow making seasonal predictions. Of course, mechanical transmission cannot be neglected (Desquesnes et al., 2009) and can lead to a risk of trypanosomosis independent from tsetse, including outside the tsetse belt (Pagabeleguem et al., 2012). Moreover, high temperature might have contradictory impacts on different factors of transmission, for example by reducing tsetse lifespan and density (Van den Bossche et al., 2010).

In our study, it was surprising that air temperature measured from meteorological stations located quite far from the study sites better predicted tsetse infection rates than the MODIS LSTd measured in the $1^{*} 1 \mathrm{~km}$ pixels where the tsetse were captured. This is probably due to discrepancies between LSTd and air temperature, in relation to land cover, whereas the maximum air temperatures in the meteorological stations were probably highly correlated to those of the study sites. It would be very interesting to conduct a similar study including the monitoring of air temperature and hygrometry using field meteorological stations. In our study, the correlation between LSTd and max temperature was very high $\left(r^{2}=0.77, p<10^{-3}\right)$. LSTd was still highly correlated to the

\section{REFERENCES}

Akoda, K. (2009). Effect of Nutritional Stress on the Tsetse Fly's Vector Competence and its Implications on Trypanosome Transmission in the Field. Ph.D. Faculty of Veterinary Medicine, Ghent University.

infection rate, even if less powerful than max temperature to predict it. LSTd is a MODIS composite synthetic variable. In Senegal, we, however, observed important discrepancies between air temperature measured on the ground and LSTd. Other indexes like NDVI and MIR could be combined to the latter to better predict air temperature, with an accuracy of $\sim 1^{\circ} \mathrm{c}$. Actually, these parameters are correlated to land cover, which in turn impacts air temperature. Efforts are presently underway in Senegal to build models of air temperatures using a network of field meteorological stations. The inverse relationship observed with monthly rainfalls in the case of $T$. congolense might be due to a mitigation of the evapotranspiration caused by high temperatures by an increase of relative hygrometry. Actually, tsetse survive longer at high temperatures when relative hygrometry is higher (Buxton, 1955).

A recent model predicted a large-range expansion of sleeping sickness in East and South Africa, caused mainly by a shift of up to 60 per cent of its geographical extent (Moore et al., 2012). This change was mainly anticipated from predicted changes in global temperatures impacting on the following entomological and epidemiological parameters: total tsetse population size, natural death rate of tsetse, parasite maturation rate in tsetse and tsetse biting rate. However, the potential impact of temperature on tsetse primary infection rate was not considered and might improve greatly model predictions. The raw data generated in this survey are made available to encourage modeling efforts (Supplementary file 1). Trap locations can also be requested from the corresponding author.

\section{ACKNOWLEDGMENTS}

This work benefited from international collaboration through the Centre International de Recherche-Développement sur l'Elevage en Zone Subhumide (CIRDES) and the Centre de. Coopération Internationale en Recherche Agronomique pour le Développement (CIRAD). It was carried out with the support of the Fragfly Wellcome Trust (Project 075824; http:// wt-fragfly.cirad.fr/). We are indebted to the director general of CIRDES, Professor Abdoulaye Gouro for the provision of excellent working conditions, and to Adama Sana, Bila Cene. Félix Sanou and Lansina Sanogo for assistance during the field studies. Meteorological data were kindly provided by weather stations [Agence pour la Sécurité de la Navigation Aérienne en Afrique et à Madagascar (ASECNA)] at Dédougou and Bobo-Dioulasso and MODIS processed data by the Department of Zoology of the University of Oxford.

\section{SUPPLEMENTARY MATERIAL}

The Supplementary Material for this article can be found online at: http://www.frontiersin.org/Cellular_ and_Infection_Microbiology/10.3389/fcimb.2013.00047/abstract

J. Med. Entomol. 46, 1446-1449. doi: 10.1603/033.046.0629

Akoda, K., Van den Bossche, P., Marcotty, T., Kubi, C., De Deken, R., and Van den Abbeele, J. (2009b). Nutritional stress affects the tsetse fly's immune gene expression.
Med. Vet. Entomol. 23, 195-201. doi: $\quad 10.1111 /$ j.1365-2915.2009.0 0799.x

Coosemans, M., et al. (2009。 Maturation of a Trypanosoma brucei infection to the infectious metacyclic stage is enhanced in nutritionally stressed tsetse flies.
Bates, D., Maechler, M., and Bolker, B. (2011). "Lme4: Linear Mixed-eects Models Using S4 Classes, R Package Version 0.999375-40/r1308." 
Bouyer, J., Balenghien, T., Ravel, S., Vial, L., Sidibé, I., Thévenon, S., et al. (2009). Population sizes and dispersal pattern of tsetse flies: rolling on the river. Mol. Ecol. 18, 2787-2797. doi: 10.1111/j.1365294X.2009.04233.x

Bouyer, J., Guerrini, L., César, J., De La Rocque, S., and Cuisance, D. (2005). A phyto-sociological analysis of the distribution of riverine tsetse flies in Burkina Faso. Med. Vet. Entomol. 19, 372-378. doi: 10.1111/j.13652915.2005.00584.x

Bouyer, J., Guerrini, L., Desquesnes, M., de la Rocque, S., and Cuisance, D. (2006). Mapping african animal trypanosomosis risk from the sky. Vet. Res. 37, 633-645. doi: 10.1051/vetres:2006025

Bouyer, J., Ravel, S., Vial, L., Thévenon, S., Dujardin, J.-P., de Meeus, T., et al. (2007). Population structuring of Glossina palpalis gambiensis (Diptera: Glossinidae) according to landscape fragmentation in the Mouhoun river, Burkina Faso. J. Med. Entomol. 44, 788-795. doi: 10.1603/0022258544[788:PSOGPG]2.0.CO;2

Burnham, K. P., and Anderson, D. R. (2002). Model Selection and Multimodel Inference: A Practical Information-Theoretic Approach. New-York, NY: Springer-Verlag.

Burtt, E. D. (1946). Incubation of tsetse pupae: increased transmission rate of Trypanosoma rhodesiense in Glossina morsitans. Ann. Trop. Med. Parasitol. 40, 18-28.

Buxton, P. A. (1955). The Natural History of Tsetse Flies. An Account of the Biology of the Genus Glossina (Diptera). London: Lewis H. K. and Co Ltd.

Cuisance, D., Février, J., Dejardin, J., and Filledier, J. (1985). Dispersion linéaire de Glossina palpalis gambiensis et $G$. tachinoides dans une galerie forestière en zone soudanoguinéenne (Burkina Faso). Rev. Elev. Méd. Vét. Pays Trop. 38, 153-172.

Desquesnes, M., Biteau-Coroller, F., Bouyer, J., Dia, M. L., and Foil, L. D. (2009). Development of a mathematical model for mechanical transmission of trypanosomes and other pathogens of cattle transmitted by tabanids. Int. J. Parasitol. 39, 333-346. doi: 10.1016/j.ijpara.2008.07.004

Desquesnes, M., and Davila, A. M. R. (2002). Applications of PCR-based tools for detection and identification of animal trypanosomes: a review and perspectives. Vet. Parasitol. 109, 213-231. doi: 10.1016/S0304-401700270-4

Dyer, N. A., Rose, C., Ejeh, N. O., and Acosta-Serrano, A. (2013). Flying tryps: survival and maturation of trypanosomes in tsetse flies. Trends in Parasitology 29, 188-196. doi: 10.1016/j.pt.2013.1002.1003

Ford, J., and Leggate, B. M. (1961). The geographical and climatic distribution of trypanosome infection rates in Glossina morsitans group of tsetse-flies. Trans. Roy. Soc Trop. Med. Hyg. 55, 383-397. doi: 10.1016/0035-920390108-0

Guerrini, L., and Bouyer, J. (2007). Mapping African animal trypanosomosis risk: the landscape approach. Vet. Ital. 43, 643-654.

Guerrini, L., Bord, J. P., Ducheyne, E., and Bouyer, J. (2008). Fragmentation analysis for prediction of suitable habitat for vectors: the example of riverine tsetse flies in Burkina faso. J. Med. Entomol. 45, 1180-1186. doi: 10.1603/0022258545[1180:FAFPOS] 2.0.CO;2

Hurvich, C. M., and Tsai, C.-L. (1995). Model selection for extended quasilikelihood models in small samples. Biometrics 51, 1077-1084. doi: $10.2307 / 2533006$

Itard, J., Cuisance, D., and Tacher, G. (2003). "Trypanosomoses: historique - répartition géographique," in Principales Maladies Infectieuses Et Parasitaires Du Bétail. Europe et Régions Chaudes, eds P.-C. Lefevre, J. Blancou, and R. Chermette (Paris: Lavoisier), 1607-1615.

Koné, N., Bouyer, F., Vitouley, H., Sidibé, I., Ngoran, E., Vial, L., et al. (2012). Perceptions des éleveurs et stratégies de gestion du risque trypanosomien dans le bassin du fleuve Mouhoun, au Burkina Faso. Cah. Agric. 21, 404-416.

Koné, N., Bouyer, J., Ravel, S., Vreysen, M. J. B., Domagni, K. T., Causse, S., et al. (2011a). Contrasting population structures of two vectors of African trypanosomoses in Burkina Faso: consequences for control. PLoS Negl. Trop. Dis. 5:e1217. doi: 10.1371/journal.pntd.0001217

Koné, N., N'Goran, E. K., Sidibé, I., Kombassere, A. W., and Bouyer, J. (2011b). Spatio-temporal distribution of tsetse (Diptera: Glossinidae) and other biting flies (Diptera: Tabanidae and Stomoxinae) in the Mouhoun River Basin, Burkina Faso. Med. Vet. Entomol. 25, 156-168. doi: $\quad$ 10.1111/j.1365-2915.2010. 00938.x

Kubi, C., Van den Abbeele, J., DE Deken, R., Marcotty, T., Dorny, P., and Van den Bossche, P. (2006). The effect of starvation on the susceptibility of teneral and non-teneral tsetse flies to trypanosome infection. Med. Vet. Entomol. 20, 388-392. doi 10.1111/j.1365-2915.2006.00644.x

Laird, N. M., and Ware, J. H. (1982). Random-effects models for longitudinal data. Biometrics 38, 963-974. doi: $10.2307 / 2529876$

Leak, S. (1998). Tsetse Biology and Ecology. Their Role in the Epidemiology and Control of Trypanosomosis. Oxon: CABI publishing with ILRI.

Lefevre, T., and Thomas, F. (2008) Behind the scene, something else is pulling the strings: emphasizing parasitic manipulation in vector-borne diseases. Infect. Genet. Evol. 8, 504-519. doi: 10.1016/j.meegid.2007.05.008

Métras, R., Bouyer, J., Vitouley, H., Koné, N., and Lancelot, R. (2008). "Impact of landscape fragmentation and season on cattle trypanosomosis, in yhe Mouhoun river basin, Burkina Faso," in Xth European Multicolloquium of Parasitology, Paris: Société Française de Parasitologie.

Moore, S., Shrestha, S., Tomlinson, K. W., and Vuong, H. (2012). Predicting the effect of climate change on African trypanosomiasis: integrating epidemiology with parasite and vector biology. J. $R$. Soc. Interface 9, 817-830. doi: 10.1098/rsif.2011.0654

Ndegwa, P. N., Irungu, L. W., and Moloo, S. K. (1992). Effect of puparia incubation temperature: increased infection rates of Trypanosoma congolense in Glossina morsitans centralis, $G$. fuscipes fuscipes and G. brevipalpis. Med. Vet. Entomol. 6, 127-130. doi: 10.1111/j.13652915.1992.tb00588.x

Pagabeleguem, S., Sangaré, M. Bengaly, Z., Akoudjin, M., Belem, A. M. G., and Bouyer, J. (2012). Climate, cattle rearing systems and African animal Trypanosomosis risk in Burkina Faso. PLoS ONE 7:e49762. doi: 10.1371/journal. pone. 0049762

R Core Team. (2013). R: A Language and Environment for Statistical Computing. Vienna: R Foundation for Statistical Computing. Available online at: http://www.R-project.org Solano, P., Michel, J. F., Lefrançois, T., de La Rocque, S., Sidibe, I., Zoungrana, A., et al. (1999). Polymerase chain reaction as a diagnosis tool for detecting trypanosomes in naturally infected cattle in Burkina Faso. Vet. Parasitol. 86, 95-103. doi: 10.1016/S0304-401700137-5

Tran, A., Biteau-Coroller, F., Guis, H., and Roger, F. (2005). Modélisation des maladies vectorielles. Epidémiol. Santé Anim. 47, 35-51.

Van den Bossche, P., De La Rocque, S., Hendrickx, G., and Bouyer, J. (2010). A changing environment and the epidemiology of tsetsetransmitted livestock trypanosomiasis. Trends Parasitol. 26, 236-243. doi: 10.1016/j.pt.2010.02.010

Vreysen, M., Balenghien, T., Saleh, K. M., Maiga, S., Koudougou, Z. Cecchi, G., et al. (2013). Releaserecapture studies confirm dispersal of Glossina palpalis gambiensis between river basins in Mali. PLoS Negl. Trop. Dis. 7:e2022. doi: 10.1371/journal.pntd.0002022

Conflict of Interest Statement: The authors declare that the research was conducted in the absence of any commercial or financial relationships that could be construed as a potential conflict of interest.

Received: 31 May 2013; accepted: 12 August 2013; published online: 29 August 2013.

Citation: Bouyer J, Koné $N$ and Bengaly $Z$ (2013) Dynamics of tsetse natural infection rates in the Mouhoun river, Burkina Faso, in relation with environmental factors. Front. Cell. Infect. Microbiol. 3:47. doi: 10.3389/fcimb. 2013.00047

This article was submitted to the journal Frontiers in Cellular and Infection Microbiology.

Copyright (c) 2013 Bouyer, Koné and Bengaly. This is an open-access article distributed under the terms of the Creative Commons Attribution License (CC BY). The use, distribution or reproduction in other forums is permitted, provided the original author(s) or licensor are credited and that the original publication in this journal is cited, in accordance with accepted academic practice. No use, distribution or reproduction is permitted which does not comply with these terms. 